\title{
Synchronous Association of Squamous Cell Carcinoma of the Larynx and Clear Cell Renal Cell Carcinoma: A Case Report
}

\author{
Mohamed Chehbouni and Benhoummad Othmane
}

\section{ABSTRACT}

\begin{abstract}
Laryngeal squamous cell carcinomas represent the most frequent form (over $90 \%$ ) of laryngeal cancer, but their association with renal tumors is rare; the literature most often reports the synchronous association of carcinoma of the larynx with carcinoma of the upper aerodigestive tract or with bronchial carcinoma. We report the case of a 60 -year-old patient, who is followed for a clear cell carcinoma of the kidney, and in whom we made the diagnosis of a squamous cell carcinoma of the larynx. We illustrate through this observation the rarity and the epidemiological profile of this presentation with a broad review of the literature.
\end{abstract}

Keywords: synchronous tumors, multiple primary malignant tumors, laryngeal cancer, renal tumors.

\section{INTRODUCTION}

The simultaneous occurrence of multiple primary malignant tumors (MPMT) is an old notion, but rarely occurs, it is a phenomenon first described by Billroth in 1889 [1]. Urologic carcinomas are most often associated with synchronous tumors of the digestive tract, breast and lung, the laryngeal localization of a synchronous primary tumor is rare.

\section{OBSERVATION}

This is a 60-year-old patient, chronic smoker at 20 Packs/Year, weaned 5 years ago. This patient was followed for renal cell carcinoma grade 1 Fuhrmann for which he received a partial bilateral nephrectomy with a right adrenalectomy at 5-month interval between the right and left side. The patient had eight months before his admission to the ENT department persistent dysphonia for which it was consulted only after the installation of an inspiratory dyspnea, for which he was tracheotomy. A cervico-thoracic CT scan was performed, showing a tumor process of the right hemilarynx involving the three stages of the larynx (Fig. 1) without any other associated localizations of the upper aerodigestive tract (UADT). A panendoscopy under general anesthesia with biopsy led to the histological diagnosis of a moderately differentiated squamous cell carcinoma of the larynx. This laryngeal tumor was classified T4N0 in a healthy patient with a Karnofsky index of more than $70 \%$. An abdominal ultrasound completed by a uroscanner had shown a mass of the lower pole of the right kidney measuring 3.5 $\mathrm{cm} / 4.2 \mathrm{~cm}$ in relation to a renal tumor recurrence (Fig. 2).

The excretory tract, bladder and left kidney were normal in appearance. Renal function was good, and the PSA level did not exceed $1.2 \mathrm{ng} / \mathrm{ml}$.

After a multidisciplinary consultation, a right enlarged nephrectomy was recommended, its immediate postoperative effects were simple. The patient then underwent a total laryngectomy and was referred to the oncology department for further treatment.

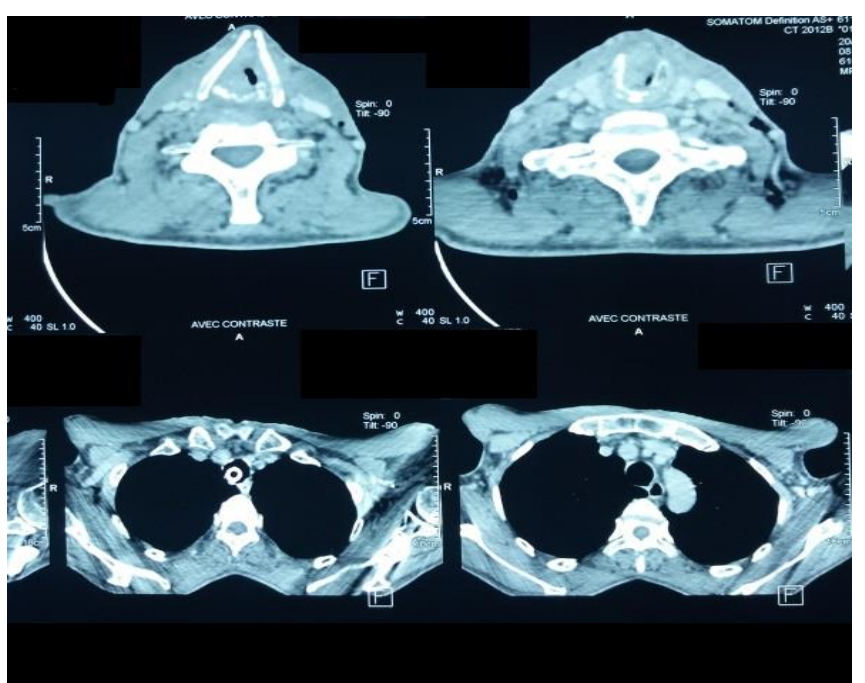

Fig. 1. CT axial sections showing the laryngeal tumor process. 


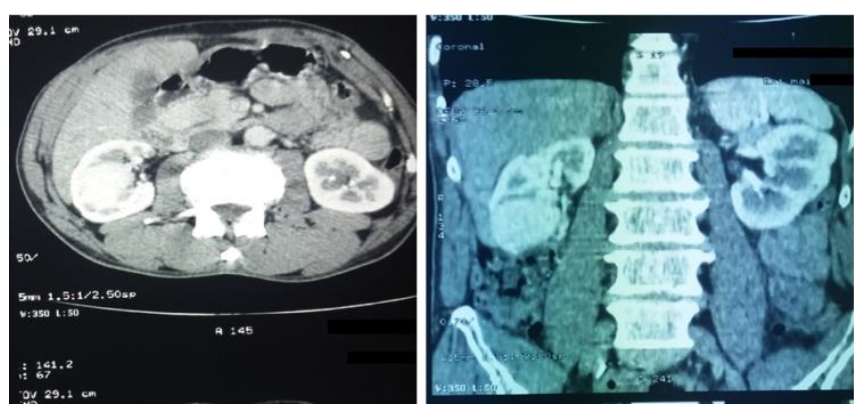

Fig. 2. axial and coronal sections of the uroscan showing the renal tumor.

\section{DISCUSSION}

The publications reporting cases of multiple primary malignancies are growing in number. The average rate of development of a second cancer across all sites is about $5 \%$ according to Moertel [2].

Warren and Gates (1932) were able to collect 40 cases of MPMT from 1078 autopsies of cancer patients, and they established the criteria for diagnosis of MPMT as follows: the existence of more than one primary tumor in different organs, or two or more primary tumors developed from different cell types within the same organ, with no notion of contiguity between the two tumors, with different metastatic pathways, so the diagnosis of metastasis must be excluded [3]. They can be synchronous or metachronous. More recently, Detterbeck et al. defined synchronous cancers as two neoplastic lesions of different histology or of the same histology provided that they are distinct and are not accompanied by lymph node or metastatic involvement when they are diagnosed less than 6 months old. each other, and metachrones when diagnosed after 6 months [4], [5].

The association of squamous cell carcinoma of the larynx with that of the UADT has been well documented in the literature; the association with renal cell carcinoma is rare. The analysis of several autopsy series has revealed $21.6 \%$ of multiple primary cancers in subjects with urological tumors [6]. These tumors were of pancreatic, esophageal, gastric, hepatic origin or localized in the bile ducts or in the gynecological area, there was no laryngeal localization [6], [7].

In a study in 1985 of 1389 new cases of laryngeal squamous cell carcinoma, Miyahara et al. were able to identify a rate of $9.94 \%$ of cases that spontaneously developed another primary synchronous or metachronous tumor, a triple association was observed in 9 cases and two cases of quadriple localization, 13 cases of metachronous cancers were induced by radiotherapy for the treatment of laryngeal carcinoma, The second location was mainly in the VADS (28.8\%), the digestive tract (44.4\%), bronchopulmonary $(15 \%)$ and urogenital $(7.8 \%)$. The urological location included the prostate and the bladder, no case of primary renal tumor associated with squamous cell carcinoma of the larynx was found in this series [8].

However, Begg et al. were able to identify a high rate of association among tumors with smoking as a risk factor, including tumors of the head and neck, kidney, lung, bladder, and esophagus [9].

Turkish women recently reported a $1.2 \%$ rate of PMT in a 10 -year study of 9772 cancer patients, and the most common synchronous tumor associations observed in men in this study were: genitourinary and gastrointestinal tumors (4\%), ENT and lung tumors (3\%), ENT and genitourinary tumors $(2 \%)$ [10]. Thus, the association of laryngeal tumor and renal tumor has not been reported in the literature to our knowledge.

The mechanisms explaining the occurrence of MPMT remain poorly elucidated, several theories are advanced: those related to age, hormonal, genetic environmental, immunological or iatrogenic, but smoking intoxication seems to play a determining role, some authors incriminate the life expectancy that has become longer and the development of diagnostic and therapeutic means, Indeed, the advent of effective anti-cancer treatments have led to a substantial cure rate with a high 5-year survival rate, but at the expense of the emergence of secondary tumors, sometimes iatrogenic to these complementary therapies [11]-[13].

In our clinical observation, our patient had a major risk factor which is smoking, the synchronous association of the renal and laryngeal tumor could be a coincidence and it could simply be a sporadic case, but the genetic character in this patient cannot be ruled out, especially since the renal involvement in him is bilateral, multifocal and recurrent, which could be part of Von Hippel Lindau's disease [14], [15].

The histological study allowed us to establish the diagnosis of a synchronous tumor, but it is always advisable to think of a metastasis in front of a second localization despite the atypical localization, 7 cases of laryngeal metastases of renal origin have been reported in the literature [16].

Therapeutically, TMMPs present a real challenge; there is no formal consensus on management given the rarity and multi-variety of these associations [17]. Few data are available regarding the treatment of these synchronous cancers. If each lesion is carcinologically resectable, the surgical approach is preferred (if the patient is operable). In our patient, we discussed in a multidisciplinary staff, a surgical removal of the renal tumor first since it has a very high metastatic power compared to the laryngeal carcinoma, the secondary therapeutic attitude will depend on the renal prognosis, a surgery or a radiotherapy associated or not with a chemotherapy will be considered thereafter for the treatment of the laryngeal carcinoma, these decisions require a thorough reasoning and a multidisciplinary consultation in order to not harm the patient's quality of life.

\section{CONCLUSION}

This case illustrates the interest of the follow-up and the realization of an extension work-up for cancer patients in order to be able to diagnose early a synchronous or metachronous tumor, because the therapeutic attitude changes according to the nature and the site of the second tumor, the therapeutic decision which remains difficult to establish, requires several speakers of different disciplines. Finally, this entity of TMMP also prompts us to launch a program of genetic study in cancer patients, a certain genetic profile could determine us the patients potentially carrying multiple tumor attacks, which would have a great impact on the therapeutic management in the future. 


\section{REFERENCES}

[1] Brown, M. Second primaries in cases of cancer of the larynx. $J$. Laryngol. Otol. 92, 991-996 (1978).

[2] Moertel, C. Multiple primary malignant neoplasms. Introduction and presentation of data. Cancer, 14: 221-230 (1961).

[3] Warren, S. \& Gates, O. Multiple primary malignant tumors: a survey of the literature and statistical study. Am J Cancer. 1358-1414 (1932).

[4] Detterbeck, F. C., Jones, D. R., Kernstine, K. H. \& Naunheim, K. S. Special Treatment Issues*. Chest 123, 244S-258S (2003).

[5] Irimie, A., Achimas-Cadariu, P., Burz, C. \& Puscas, E. Multiple Primary Malignancies - Epidemiological Analysis at a Single Tertiary Institution. 6 .

[6] Ono, Y. et al. Autopsy findings of patients with urological neoplasms. Int J Clin Oncol 7, 0301-0305 (2002).

[7] Piccinini, L., Luppi, G., Zoboli, A. \& Torricelli, P. Occasional Diagnosis of Synchronous Renal Cell Carcinoma during Staging of Other Primary Tumors. Tumori 82, 488-490 (1996).

[8] Miyahara, H., Tsuruta, Y., Umatani, K., Yoshino, K. \& Sato, T. Multiple Primary Tumors in Patients With Head and Neck Cancer. Auris Nasus Larynx 12, S30-S35 (1985).

[9] Begg, C. B., Zhang, Z., Sun, M., Herr, H. W. \& Schantz, S. P. Methodology for Evaluating the Incidence of Second Primary Cancers with Application to Smoking-relted Cancers from the Surveillance, Epidmiology, and End Results (SEER) Program. American Journal of Epidemiology 142, 653-665 (1995).

[10] Etiz, D., Metcalfe, E. \& Akcay, M. Multiple primary malignant neoplasms: A 10-year experience at a single institution from Turkey. $J$ Can Res Ther 13, 16 (2017).

[11] Mh, G., Rn, H. \& Jr, F. J. Subsequent cancer in patients with chronic lymphocytic leukemia--a possible immunologic mechanism. J Natl Cancer Inst 61, 337-340 (1978).

[12] Khuri, F. R. et al. The Impact of Smoking Status, Disease Stage, and Index Tumor Site on Second Primary Tumor Incidence and Tumor Recurrence in the Head and Neck Retinoid Chemoprevention Trial. Cancer Epidemiol Biomarkers Prev 10, 823-829 (2001).

[13] Gallo, O. \& Bianchi, S. p53 Expression: a potential biomarker for risk of multiple primary malignancies in the upper aerodigestive tract. European Journal of Cancer Part B: Oral Oncology 31, 53-57 (1995)

[14] Richard, S. Prédispositions héréditaires au cancer rénal. Actualités néphrologiques Jean Hamburger 131-150 (2006).

[15] Rae, F. K., Hooper, J. D., Nicol, D. L. \& Clements, J. A. Characterization of a novel gene,STAG1/PMEPA1, upregulated in renal cell carcinoma and other solid tumors. Mol. Carcinog. 32, 44-53 (2001).

[16] Sarkis, P., Bou-Malhab, F. \& Mouaccadieh, L. Métastase laryngée unique d'un carcinome rénal à cellules claires : cas clinique et revue de la littérature. Progrès en Urologie 22, 307-309 (2012).

[17] Luciani, A. Multiple primary malignancies. Seminars in Oncology 31, 264-273 (2004). 